\title{
DIRECT BRAIN-COMPUTER COMMUNICATION WITH USER REWARDING MECHANISM
}

\author{
Gary N. Garcia, Touradj Ebrahimi, Jean-Marc Vesin and Abel Villca. \\ Swiss Federal Institute of Technology - EPFL, CH-1015 Lausanne, Switzerland \\ Gary.garcia@epfl.ch, Touradj.Ebrahimidepfl.ch, \\ Jean-Marc.Vesin@epfl.ch, Abel.Villcadepfl.ch
}

\begin{abstract}
An electroencephalogram (EEG) based Brain Computer Interface (BCI) and its main components are presented. The BCI operation model is established on the basis of a three-state machine where the direct transitions from the neutral to the active state are not allowed. A transition state is introduced in order to make the user confirm his intents through latency. The latency is a dynamic parameter that decreases when the user achieves an acceptable level of performance.

EEG signals are classified in the space generated by their correlative time-frequency-space representation (CTFSR). Nonlinear separation in this space is achieved by using the support vector machine approach. An adequate kernel design makes the computations take place in the temporal domain.

Good efficiency is obtained after five experiment sessions by two subjects.
\end{abstract}

\section{INTRODUCTION}

Systems capable of understanding the different facets of human communication and interaction with computers are among trends in Human-Computer Interfaces (HCI).

An HCI which is built on the guiding principle (GP): "think and make it happen without any physical effort" is called a brain-computer interface (BCI). Indeed, the "think" part of the GP involves the human brain, "make it happen" implies that an executor is needed (here the executor is a computer) and "without any physical effort" means that a direct interface between the human brain and the computer is required.

To make the computer interpret what the brain intends to communicate necessitates monitoring of the brain activity. Among the possible choices the scalp recorded electroencephalogram (EEG) appears to be an adequate alternative because of its good time resolution and relative simplicity. Furthermore, there is clear evidence that observable changes in EEG result from performing given mental activities [1].
In this study, we present an EEG based BCI hereafter called BCI.

\section{BCI IN A NUTSHELL}

Our BCI system is subdivided into three subsystems, namely EEG acquisition, EEG signal processing and output generation. (Figure 1).

The EEG acquisition subsystem is composed of an electrode array arranged according to the 10-20 international system [2] and a digitization device. The acquired signals are often noisy and may contain artefacts due to muscular and ocular movements.

The EEG signal processing subsystem is subdivided into a preprocessing unit, responsible for artefact detection, and a feature extraction and recognition unit that determines the command sent by the user to the BCI. This command is in turn sent to the output subsystem which generates a "system answer" that constitutes a feedback to the user who can modulate his mental activities so as to produce those EEG patterns that make the BCI accomplish his intents.

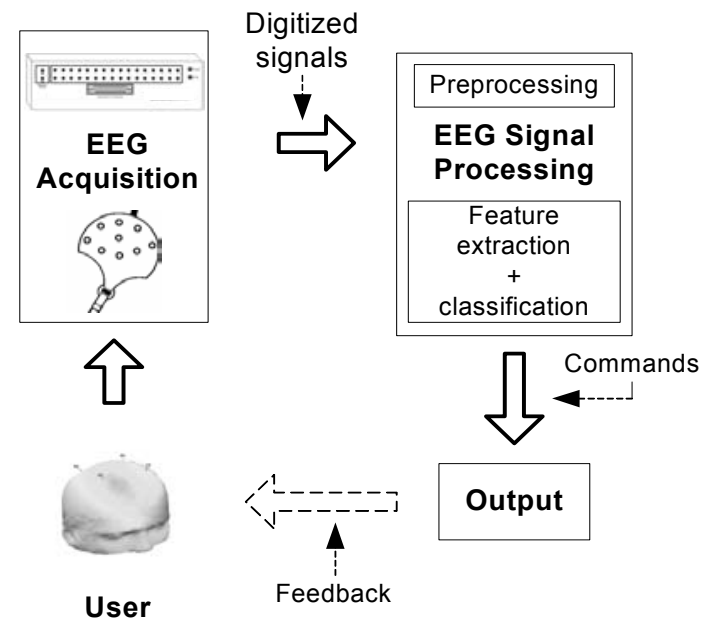

Figure 1. General BCI architecture. 
Figure 2 illustrates the basic scheduling of our BCI. The $\mathrm{BCI}$ period is the average time between two consecutive answers and the EEG trial duration is the duration of EEG that the $\mathrm{BCI}$ needs to analyze in order to generate an answer. We assume that every EEG trial elicits a system answer.

We call "neutral state" when nothing happens (the BCI provides a neutral answer), the "active state" when the BCI executes something, the "neutral EEG set" as composed of those EEG trials that elicit the neutral answer and the "active EEG set" the complement of the neutral EEG set.

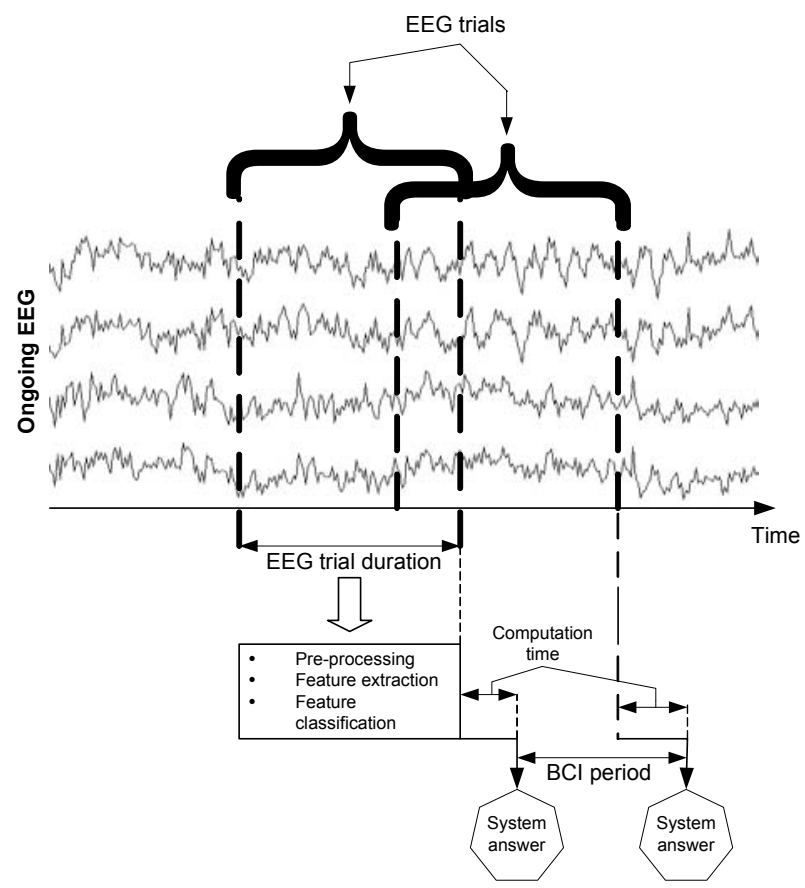

Figure 2. BCI scheduling.

The ideal BCI is a two-state machine whose state changes occur at a rate defined by the BCI period and are determined by a Boolean variable B1 (activation) which becomes true when the BCI detects an element of the active EEG set and false otherwise (Figure 3).

The ideal BCI behave properly when the recognition error rate is near zero.

In a real application, the false positive error (the system switches to the active state while the corresponding EEG trial belongs to the neutral EEG set) and the false negative error (the system switches to the neutral state while the corresponding EEG trial belongs to the active set) are not zero. Depending on the application, these errors are differently penalized.

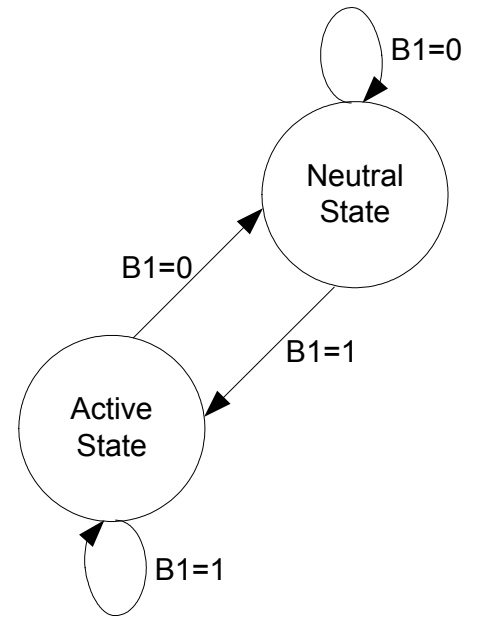

Figure 3. Ideal BCI.

We propose a less ideal $\mathrm{BCI}$ by introducing a transition state so that the BCI cannot switch from the neutral to the active state immediately. The BCI remains in the transition state as long as a second Boolean variable B2 (confirmation) is false (Figure 4). B2 is true if the $\mathbf{L}$ (latency parameter) previous EEG trials are equally recognized as the current EEG trial.

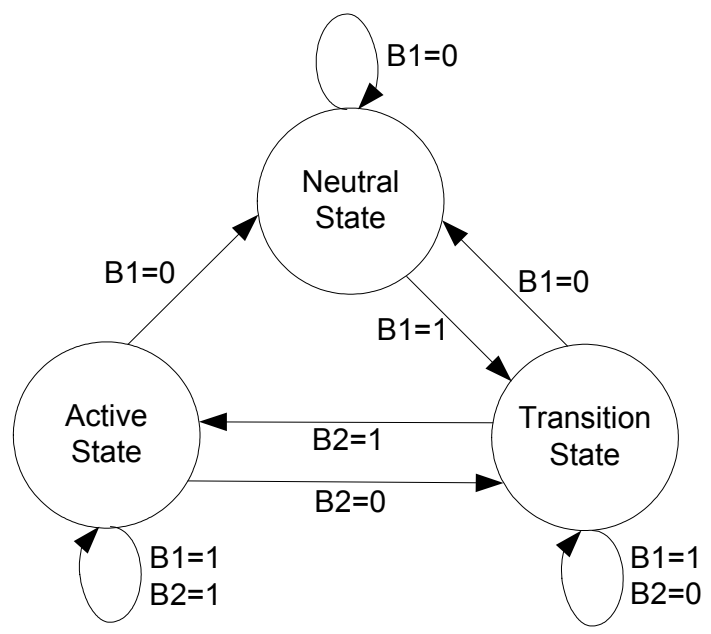

Figure 4. Less ideal BCI.

The introduction of the transition state allows us to prevent the BCI from abruptly switching to the active state and to introduce a user rewarding mechanism by reducing his latency when false positive and false negative error rates decrease.

The latest statement constitutes the key for the computation of $\mathbf{L}$. Let, $p_{+\mid+}$be the true positive recognition rate and $p_{+\mid-}$be the false positive error rate. Then, $\mathbf{L}$ increases if $p_{+\mid-}$increases or $p_{+\mid+}$decreases. Likewise $\mathbf{L}$ decreases if $p_{+\mid-}$decreases or $p_{+\mid+}$increases. 
The optimal value $(\mathbf{L}=0)$ is reached when $p_{+++}$is 1 and $p_{+\mid-}$is 0 . Thus, $\mathbf{L}$ can be calculated as

$$
\mathbf{L}=\operatorname{nint}\left(-C_{L} \log \left(p_{+++}\left(1-p_{++-}\right)\right)\right)
$$

where $\operatorname{nint}(\bullet)$ is the nearest integer function and $C_{L}$ is a normalization constant.

In practice, for the sake of user comfort the value of $\mathbf{L}$ multiplied by the BCI period should not exceed two seconds.

The BCI parameters are summarized in Table 1. Signal processing parameters are described in Section 3.

The optimal values for the BCI parameters are determined in the training phase. However, they should be continuously updated in order to take into account possible variations in the EEG caused by different brain's background activities over time. Thus, BCI operation requires constant training and adaptation from both, the user and the computer [3].

\begin{tabular}{|l|}
\hline BCI period \\
\hline EEG trial duration \\
\hline Latency \\
\hline Signal processing parameters \\
\hline
\end{tabular}

Table 1. BCI parameters.

\section{EEG SIGNAL PROCESSING IN BCI APPLICATIONS}

\subsection{EEG trial preprocessing}

The preprocessing aims at identifying those EEG trials contaminated by noise. By definition (Sect. 2) a noisy EEG trial belongs to the neutral EEG set.

External electromagnetic perturbations, muscular and ocular movements are the main sources of noise.

External perturbations are essentially present at high frequencies (above $40 \mathrm{~Hz}$ ). Since the interesting EEG frequencies are located below this limit, each EEG trial is filtered between 1 and $40 \mathrm{~Hz}$.

Muscular movements induce relatively high frequency (above $25 \mathrm{~Hz}$ ), high amplitude components (muscle artefacts) in electrodes located in the motor area [4]. We set a power threshold (determined during the training phase) on the frequency band above $25 \mathrm{~Hz}$, in order to detect such artefacts.

Ocular artefacts are identified by tracking the abrupt power changes in the signals recorded at prefrontal electrodes

\subsection{Feature space}

In the context of BCI, the features used for EEG trial classification mainly result from the time, frequency, and time-frequency analysis.

Most of the research groups work in the frequency domain and extract the information characterizing mental activities from the nonparametric and parametric spectral representations of EEG [3]. Also, the joint spectral properties of the EEG components are analyzed in [5] for detecting particular emotional states.

The relationship between the time evolutions of the signals coming from different electrodes serves as an indication of motor activities in [6]. Useful information can also be extracted from particular brain configurations that can be interpreted in terms of brain states [7][8].

Time-frequency and time-scale representations of EEG signals were exploited for finding those neuronal groups that synchronize their activity as a response to a particular stimulus (event related potentials) [9][10].

From the above considerations it can be stated that mental activities, when mapped onto the time-frequency representation of EEG signals, display a picture that illustrates the cooperative activity of neuronal groups. A possible way to explore this activity consists in analyzing the joint time-frequency-space correlations between the components of an EEG signal.

The correlative time-frequency-space representation $(\mathrm{CTFSR})$ of an EEG trial $X(t)=\left[\begin{array}{lll}x_{1}(t) & \ldots & x_{N}(t)\end{array}\right]^{\mathbf{T}}(\mathbf{T}$ is the transpose operator) composed of $N$ signals measured at different electrodes, is defined as [11]

$$
A_{X}(\theta, \tau)=\int X\left(t+\frac{\tau}{2}\right) \cdot X^{\mathbf{H}}\left(t-\frac{\tau}{2}\right) e^{j \theta t} d t
$$

where $\theta$ and $\tau$ are the frequency and time lags respectively, "." is the ordinary matrix multiplication and ${ }^{\mathbf{H}}$ is the Hermitian operator.

A measure of the similarity between the CTFSR of two EEG trials $X(t)$ and $Y(t)$ is given by

$$
D\left(A_{X}, A_{Y}\right)=\mathbf{1}_{N} \cdot \iint \Delta_{(X, Y)}(\theta, \tau) \odot \Delta_{(X, Y)}^{*}(\theta, \tau) d \theta d \tau \cdot \mathbf{1}_{N}^{\mathbf{T}}
$$

where $\odot$ is the element wise matrix multiplication, $*$ is the complex conjugate operator, $\mathbf{1}_{N}$ is a $1 \mathrm{x} N$ real matrix with unit components and

$$
\Delta_{(X, Y)}(\theta, \tau)=A_{X}(\theta, \tau)-A_{Y}(\theta, \tau)
$$

We define an inner product in the CTFSR space as follows.

$$
\left\langle A_{X}, A_{Y}\right\rangle=\mathbf{1}_{N} \cdot \iint A_{X}(\theta, \tau) \odot A_{Y}^{*}(\theta, \tau) d \theta d \tau \cdot \mathbf{1}_{N}^{\mathbf{T}}
$$

The inner product in Eq. (5) can be directly calculated in the time domain. 


$$
\left\langle A_{X}, A_{Y}\right\rangle=\mathbf{1}_{N} \cdot \iint X(t) \cdot X^{*}(t) \odot Y^{*}(t) \cdot Y(t-\tau) d t d \tau \cdot \mathbf{1}_{N}^{\mathrm{T}}(6)
$$

Since the Eq. (3) can be written in terms of inner products (Eq. (7)), the similarity measure in the CTFSR space can be directly calculated in the time domain.

$$
D\left(A_{X}, A_{Y}\right)=\left\langle A_{X}, A_{X}\right\rangle+\left\langle A_{Y}, A_{Y}\right\rangle-\left\langle A_{X}, A_{Y}\right\rangle-\left\langle A_{Y}, A_{X}\right\rangle
$$

\subsection{Classification in the CTFSR space}

The classification in the CTFSR space is a nontrivial problem because of the large dimensionality of this space. Common approaches in the univariate case $(N=1)$ suggest to enhance the discriminating regions by multiplying the CTFSR by a time-frequency kernel (TFK) [12]. In the multivariate case a linear Fisher discriminator was used in [13] and multivariate TFKs were proposed in [14]. However, considerable computational effort was necessary for obtaining acceptable results.

We applied the support vector machine (SVM) approach for classification in the CTFSR space. The advantage of using SVMs resides in the fact that SVM computations only involve inner products [15]. As the inner product in Eq. (6) is computed in the time domain, we do not need to explicitly calculate the CTFSR of EEG trials.

In addition, one often points out the good generalization capabilities of SVM based classifiers.

For our purposes we consider only the two-class classification problem. Multi-class classification can be done with multiple pair-wise comparisons.

The SVM approach for two-class classification can be summarized as follows (for a more detailed explanation the reader is referred to [15]).

Given a set of decision functions parameterized by $\alpha \in \Lambda$ ( $\Lambda$ is a space $)$

$$
\left\{f_{\alpha}\right\}_{\alpha \in \Lambda}, f_{\alpha}: \text { CTFSR space } \rightarrow\{-1,+1\}
$$

and a labeled training set $\Gamma=\left\{\left(A_{X_{i}}, y_{i}\right) ; 1 \leq i \leq M\right\}$ where $y_{i} \in\{-1,+1\}$ is the label of the EEG trial $X_{i}$ and $A_{X_{i}}$ the CTFSR of $X_{i}$, we wish to find a function $f_{\alpha^{\prime}}$ that minimizes the risk of misclassification of new unknown EEG trials, given by the risk functional

$$
R(\alpha)=\frac{1}{2} \int\left|f_{\alpha}\left(A_{X}\right)-y\right| d P\left(A_{X}, y\right)
$$

where $P\left(A_{X}, y\right)$ is the unknown probability distribution that generated $\Gamma$.

One can show that the risk functional is bounded by the sum between the empirical error (depending on the training set) and a complexity measure of the set of functions $\left\{f_{\alpha}\right\}[15]$.
In the linear separable case the optimal decision function is a hyperplane that can be written as

$$
f\left(A_{X}\right)=\operatorname{sgn}\left(\left\langle w, A_{X}\right\rangle+b\right)
$$

The optimal estimates for $w$ and $b$ are found by solving

$$
\begin{aligned}
& (w, b)=\underbrace{\arg \min }_{w, b}\left[\frac{1}{2}\langle w, w\rangle-v \rho+\mathrm{R}_{\mathrm{e} m p}\right] \\
& \mathrm{R}_{\mathrm{e} m p}=\frac{1}{M} \sum_{i=1}^{M} \max \left\{0, \rho-y_{i} \cdot f\left(A_{X_{i}}\right)\right\}
\end{aligned}
$$

where $\rho$ (loss parameter) and $v$ are user defined constants. The support vectors (SVs) are the $A_{X_{i}}$ 's for which $y_{i} \cdot f\left(A_{X_{i}}\right) \leq \rho$. It can be shown that $v$ is a lower bound on the fraction of training elements that are SVs [15].

The results in [15] show that $w$ is a linear combination of the $A_{X_{i}}$ 's

$$
w=\sum_{i=1}^{M} \alpha_{i} y_{i} A_{X_{i}}
$$

and that solving Eq. (9)is equivalent to finding

$$
\max \left[-\frac{1}{2} \sum_{i_{1}, i_{2}=1}^{M} \alpha_{i_{1}} \alpha_{i_{2}} y_{i_{1}} y_{i_{2}}\left\langle A_{X_{i_{1}}}, A_{X_{i 2}}\right\rangle\right]
$$

subject to constraints

$$
\begin{aligned}
& \sum_{i=1}^{M} y_{i} \alpha_{i} \\
& 0 \leq \alpha_{i} \leq \frac{1}{M} ; 1 \leq i \leq M \\
& \sum_{i=1}^{M} \alpha_{i} \geq v
\end{aligned}
$$

The offset $b$ and the loss parameter $\rho$ can be found using

$$
y_{i}\left(\left\langle w, A_{X_{i}}\right\rangle+b\right)=\rho \quad \text { when } \quad 0<\alpha_{i}<\frac{1}{M}
$$

The problem of nonlinear decision boundaries is solved by mapping the training set into a high dimensional space (HDS) where the decision function is linear. This mapping is done by the means of kernel functions (functions that define an inner product in the HDS). The above considerations hold in the HDS and Eqs. (8)-(13) can be applied by replacing the inner products by the kernel function.

A popular kernel function is the Radial Basis Kernel that can be written as function of inner products in the CTFSR space (Eq. (14)).

$$
K_{R B F}\left(A_{X}, A_{Y}\right)=\exp \left(-\frac{D\left(A_{X}, A_{Y}\right)}{\sigma^{2}}\right)
$$

where $\sigma^{2}$ is the kernel parameter. 
This approach allows us to perform nonlinear classification in the CTFSR space whereas the computations are done in the time domain.

As mentioned in Sect. 2, a key requirement in BCI applications consists in the regular adaptation of the classifier to the possible changes in user's mental activities. As only the SVs determine the classification parameters ( $w$ and $b$ ) we can easily update them by including the old SVs in a new training set.

\section{RESULTS AND DISCUSSIONS}

Two male right-handed subjects (S1 and S2) participated in five experiment sessions. The signals at electrodes: Fp1, Fp2, C1, C3, C4, C2, T3, T4, P3 and P4 of the 10-20 International System [2] were measured.

Three types of mental activities (MAs) were used: imagination of left (MA1) or right (MA2) index finger movements and a baseline (MA3) where the subject can imagine anything except MA1 or MA2. MA1 and MA2 are the elements of the active EEG set (Sect. 2).

Each session was preceded by a short calibration period in which the BCI was adjusted to the subject and the thresholds for the detection of artefacts were set (Sect. 3.1). During this operation, the subject could see a representation of his EEG signals mapped onto a $2 \mathrm{D}$ or $3 \mathrm{D}$ scene in order to get familiarized with the system.

The first session was entirely devoted to training. In the next sessions the first twenty minutes, after the calibration were spent in training and the remaining time (about ten minutes) in freely moving a cursor on a computer screen, using the less ideal state machine of Sect. 2.

In the training time of sessions two to five, continuous feedback was provided to the subjects indicating if the MA they were requested to perform was successfully recognized or not. The feedback of a session was provided using the updated classification parameters of the precedent session.

After the first session, the BCI period and the EEG trial duration were chosen among three possible alternatives depending on the classification error (Table 2). Based on these results the BCI period and the EEG trial duration were set to 250 and 500 milliseconds respectively for both users.

\begin{tabular}{|l|c|c|c|}
\hline & $\mathbf{0 . 1 2 s} / \mathbf{0 . 2 5 s}$ & $\mathbf{0 . 2 5 s} / \mathbf{0 . 5 s}$ & $\mathbf{0 . 5 s} / \mathbf{1 s}$ \\
\hline Subject 1 & $38 \%$ & $36 \%$ & $43 \%$ \\
\hline Subject 2 & $35 \%$ & $30 \%$ & $45 \%$ \\
\hline
\end{tabular}

Table 2. Classification error rate in the first training session for different values of BCI period/EEG trial duration

In Figure 5, we report the true and false positives rates of the last four experiment sessions (ES).

For subject 2 the true positives rate increased over the sessions reaching $88 \%$ at the end of the fifth session. The false positive rate slightly increased in the fourth session and decreased in the last session reaching $19 \%$.

At the end of the fifth session, subject 1 reached $80 \%$ and $22 \%$ for the true positive and false positive rates respectively. A decrease in the true positive rate and a slight increase in the false positive rate were observed at the end of the fourth session.

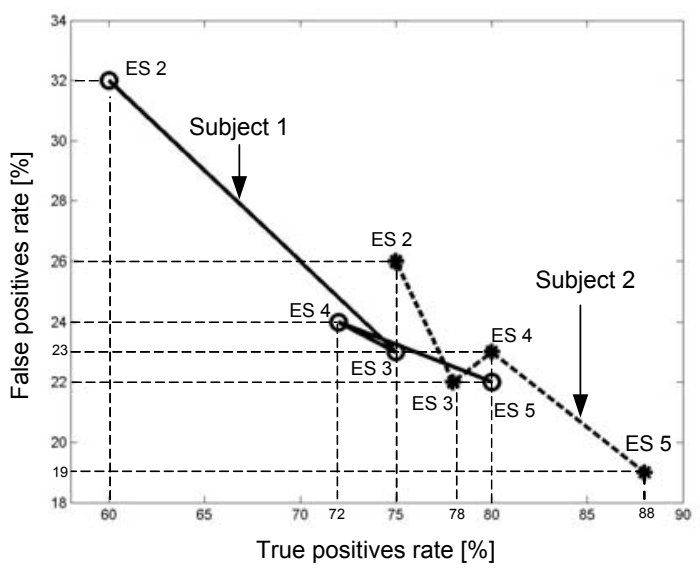

Figure 5. Evolution of the false positives vs. true positives rate for both subjects.

Interestingly, both subjects reached their best performance at the end of the fifth session. More sessions are then necessary in order to see if they can still improve their ability to operate the BCI.

In Table 3 we report the evolution of the latency parameter $\mathbf{L}$. At the end of the first session $\mathbf{L}$ was set to 4 (i.e. 4 confirmation trials) for both subjects.

\begin{tabular}{|l|c|c|c|c|}
\hline & Session 2 & Session 3 & Session 4 & Session 5 \\
\hline S1 & 4 & 3 & 3 & 2 \\
\hline S2 & 3 & 2 & 2 & 2 \\
\hline
\end{tabular}

Table 3. Evolution of the latency parameter $\mathbf{L}$ from sessions two to five.

Because of the logarithm in the definition of $\mathbf{L}$ (Eq. (1)). This parameter is not sensitive to small variations on the true positive and false positive rates. The evolution show that the results obtained were good for both subjects as $\mathbf{L}$ never increased.

In the last session we used an $\mathbf{L}$ equal to two for both subjects. This value is reasonably good in terms of user comfort because he does not need to confirm his intents for more than two BCI periods.

Using this parameter as a measure of user performance we could reward both users as they improved their ability to operate the BCI. 


\section{CONCLUSIONS}

In this paper, we defined several concepts that aim at establishing a BCI development framework.

Some of the most important parameters of a BCI were introduced and the BCI operation was characterized in terms of states that take into account the errors in the classification of EEG trials.

A way of rewarding the user improvement in using the $\mathrm{BCI}$ was proposed by updating the latency parameter.

The latency is not sensitive to small changes in the true positive recognition and false positive error rates. This constitutes a suitable behavior for a system interacting with human users.

We also presented an SVM based classification technique in the CTFSR space which performs nonlinear separation whereas all the computations are done in the temporal domain. The explicit computation of the CTFSR of EEG trials is thus avoided.

Another advantage of the SVM approach resides in the fact that the classification parameters can be easily updated by adding the old support vectors in a new training set. The requirement of continuous adaptation of the computer to the user is thus fulfilled.

In order to design an application requiring two dimensional control, at least five MAs should be recognized by the BCI. We need to explore the nature of these MAs from both user and computer perspective. Further adjustments to the BCI parameters might be necessary.

Since part of the successful operation of a BCI depends on the user himself the feedback strategy needs to be carefully designed by taking into account physiological and psychological aspects.

\section{REFERENCES}

[1] A. Gevins, "The future of electroencephalography in assessing neurocognitive functioning," $E E G$. and Clin. Neurophysiol., vol. 106, pp. 165-172, 1998.

[2] H.H. Jasper, "The Ten-Twenty electrode system of the international federation," EEG and Clin. Neurophysiol., vol. 10, pp. 371-375, 1958.

[3] J.R. Wolpaw, et al, "Brain-computer interfaces for communication and control," Clin. Neurophysiol., vol. 113, pp. 767-791, 2002.

[4] M. van de Velde, et al, "Detection of muscle artefact in the normal human awake EEG", Electroenceph. Clin. Neurophysiol., 107, pp. 149-158, 1998.

[5] A. Choppin, "Emotion Expression with Neural Networks," M. Sc. Thesis Dept. Inf. Proc. Tokyo Institute of Technology, Japan, 2000.

[6] A.B. Barreto, et al, "Spatio-temporal EEG patterns associated with voluntary motion preparation," IEEE Int. Conf. Eng. in Med. and Biol. Soc., vol. 2, pp. 857-858, 1995.

[7] E. Haselsteiner and G. Pfurtscheller, "Using TimeDependent Neural Networks for EEG Classification," IEEE. Trans. Rehab. Eng., vol. 8, no. 4, pp. 457-463, Dec. 2000.

[8] B. Obermaier, et al, "Hidden Markov models for online classification of single trial EEG data," Pattern Recog. Lett., 22, pp. 1299-1309, 2001.

[9] Chang Su Ryu, et al, "A Time-Frequency Analysis of the EEG Evoked by Negative and Positive Visual Stimuli," IEEE Int. Conf. Eng. in Med. and Biol. Soc., vol. 20, no. 4 , pp. 2012-2015, 1998.

[10]P.J. Durka, et al, "Time-Frequency Analysis of Stimulus-Driven EEG Activity by Matching Pursuit," IEEE Int. Conf. Eng. in Med. and Biol. Soc., pp. 1009-1010, 1996.

[11]M.G. Amin, et al, "The Spatial Ambiguity Function and Its Applications," IEEE Sig. Proc. Lett., vol. 7, no. 6, pp. 138-140, 2000.

[12] M. Davy and C. Doncarli, "Optimal kernels of timefrequency representations for signal classification," IEEE Int. Conf. Time-Freq. and Time-Scale Anal., pp. 581-584, 1998.

[13] G. Garcia, T. Ebrahimi and J-M. Vesin, "Classification of EEG signals in the ambiguity domain for brain computer interface applications," IEEE Int. Conf. Dig. Signal Proc., vol. 1, pp. 301-305, 2002.

[14] G. Garcia and T. Ebrahimi, "Time-frequency-space kernel for single EEG-trial classification," Nordic Signal Proc. NORSIG, 2002.

[15]B. Schölkopf and A. Smola, Learning with Kernels, MIT Press, 2002. 